
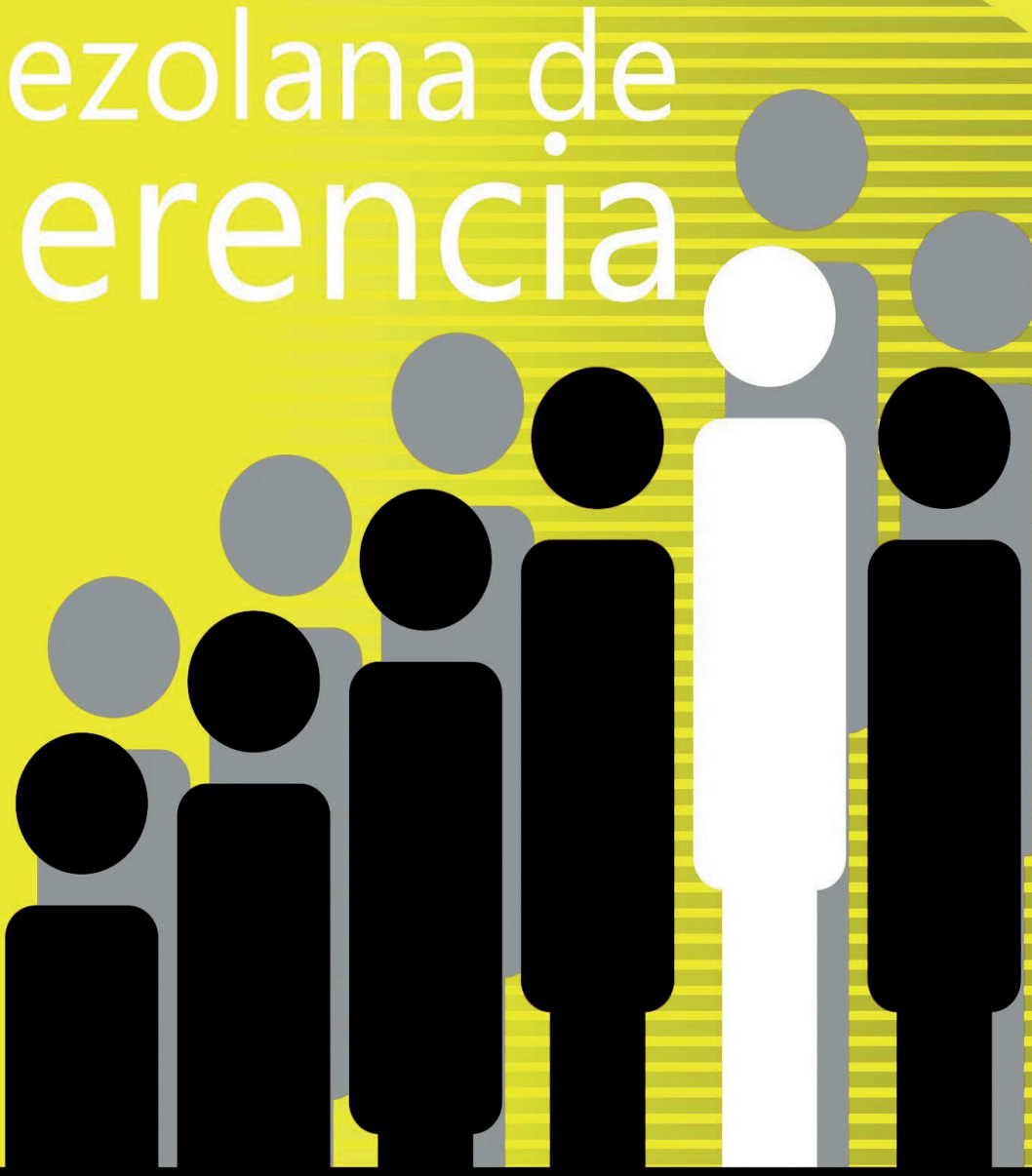


\title{
Prácticas de liderazgo dirigido en pymes del sector construcción. Propiedades psicométricas en la medición
}

\author{
Quijano García, Román Alberto* \\ Magaña Medina, Deneb Elí
}

\section{Resumen}

La industria de la construcción es un referente económico, ya que refleja de manera inmediata el desarrollo o crisis financiera de los países, la permanencia de las empresas pymes que participan en el sector es fundamental ya que proveen empleo a la población con menos preparación académica, por lo tanto, se requiere información sobre la influencia del estilo de liderazgo en las actividades de este tipo de organizaciones y su incidencia en el posicionamiento actual en el mercado. Este trabajo tiene como principal objetivo la validación psicométrica de una subescala denominada Liderazgo Dirigido obtenida del Inventario de Prácticas de Liderazgo de Kouzes y Posner (1996), así como determinar las diferencias por sector económico. El estudio es cuantitativo con un diseño no experimental transversal, los resultados de fiabilidad y validez (análisis factorial exploratorio y confirmatorio) muestran un adecuado ajuste. Se encontraron diferencias significativas entre los sectores económicos cuyo tamaño del efecto para ambas dimensiones se puede considerar moderado. Se concluye que el modelo se ajusta empíricamente a la teoría y aporta una escala válida y confiable para su empleo en el contexto estudiado.

Palabras clave: sector construcción; pymes; liderazgo dirigido; análisis factorial confirmatorio

Recibido: 20.09.20 Aceptado: 15.02.21

* Doctor en Gestión Estratégica y Políticas de Desarrollo, profesor investigador de tiempo completo en la Universidad Autónoma de Campeche, México. correo electrónico rq6715@ hotmail.com ORCID http://orcid.org/0000-0001-7316-1997

** Doctora en Ciencias Administrativas: Gestión Socioeconómica, profesora investigadora de tiempo completo en la Universidad Juárez Autónoma de Tabasco, México. correo electrónico deneb_72@yahoo.com ORCID http://orcid.org/0000-0002-8579-596x 


\title{
Exemplary leadership practices in construction sector smes. Measurements' psychometric properties
}

\begin{abstract}
The construction sector is an economic benchmark because it immediately reflects a country's development or financial crisis, the permanence of the SME's that participate in this industry is fundamental since they provide employment to the population with less academic levels completed, therefore it is required information about the influence of the leadership style in the activities of this type of organization and its incidence in the current market positioning. This research's main objective is the psychometric validation of a subscale called Exemplary Leadership obtained from Kouzes and Posner's Leadership Practices Inventory, as well as determining the differences by economic sector. The study is quantitative with non-experimental transversal design, the results of reliability and validity (exploratory and confirmatory factor analysis) show an adequate fit. Significant differences were found among the economic sectors whose effect size for both dimensions can be considered as moderated. It is concluded that the model empirically fits the theory and provides a valid and reliable scale for its use in the studied context.
\end{abstract}

Key Words: Construction sector; SME's; Exemplary Leadership; Confirmatory Factor Analysis.

\section{Introducción}

El Plan Nacional de Desarrollo de México (PND, 2019-2024), y el Plan Estatal de Desarrollo de Campeche (PED, 2019-2021), consideran prioritaria la industria de la construcción y a través de sus ejes y estrategias plantean el impulso a las actividades con potencial económico, social y ambiental, destacando la modernización de la infraestructura portuaria, aeroportuaria y ferroviaria para conectar a las regiones marginadas del país, mediante el desarrollo de mega proyectos de vías de comunicación para el transporte de bienes y servicios.

La importancia de la industria de la construcción se basa en su contribución a la economía de los países, en el caso de México, su participación en el Producto Interno Bruto fue de $6.39 \%$ al cierre del cuarto trimestre del año 2019 por encima del sector servicios financieros $(4.7 \%)$ y debajo de transporte, correos y almacenamiento $(6.49 \%)$; de igual manera se reconoce su aportación a la generación de empleos de $7.59 \%$ superior a la aportación del sector de servicios profesionales $y$ financieros $(7.03 \%)$ e inferior al sector de servicios sociales (7.84\%), 
según datos del Instituto Nacional de Estadística y Geografía (INEGI, 2020). Cabe destacar que a Junio de 2019 el estado de Campeche fue la entidad con mayor participación de obra pública con $96.1 \%$, seguido de Oaxaca con $75.8 \%$ y el Estado de México con $69.3 \%$, de acuerdo con la información del Centro de Estudios del Sector de la Construcción (CEESCO, 2020).

Con base en los planes de desarrollo económico (federal y estatal), es posible establecer como prioridad que el sector construcción esté integrado por empresas que identifiquen aquellos factores que afecten su planeación a largo plazo bajo un liderazgo dirigido y proactivo, conscientes de los retos de una economía en constante evolución. Estas organizaciones deben contar con elementos administrativos, técnicos y financieros suficientes para ser parte de los planes de desarrollo de infraestructura propuestos en los documentos rectores de la economía nacional y que puedan permanecer de forma sostenible en el mercado.

Es vasta la literatura desarrollada sobre liderazgo en aspectos como innovación (Turbay, 2013; Contreras y Barbosa, 2013a), su influencia en el recurso humano en los sectores turístico e industrial (Zayas, 2011; Contreras y Juárez, 2013c) y sus estilos en empresas de servicios (Velázquez, 2005); no así de forma específica para el sector construcción, por lo tanto, se requieren estudios que aporten conocimiento sobre el impacto que tiene éste en la toma de decisiones, la cultura organizacional, gestión del conocimiento entre otros aspectos. El objetivo del estudio fue la validación psicométrica de una subescala de elaboración propia que se retoma del Inventario de Prácticas de Liderazgo de Kouzes y Posner (1996), identificada como Liderazgo Dirigido, y que se aplicó en pequeñas y medianas organizaciones de la iniciativa privada del Sureste de México.

\section{Elementos teóricos de liderazgo}

El mundo actual requiere la función del liderazgo en cualquier tipo de organización ya sea sociedades, grupos humanos o países, Terrazas (2015) analizó las características que debe tener un líder como son: previsor, visionario, conciliador, decisor y motivador para transformar positivamente el ámbito donde realiza sus funciones, mediante un modelo tridimensional basado en las premisas ciencia y espiritualidad que son la base para el desarrollo de las aptitudes, actitudes y acciones de un líder que cuenta con conocimientos, integridad, determinación y responsabilidad para proponer con éxito cambios en su entorno.

Contreras y Castro (2013b), exponen que el poder y liderazgo son fenómenos que se presentan al interior de las organizaciones como parte de la interacción del elemento humano que colabora en la administración y operación de las mismas; a través de un análisis teórico señalan que el liderazgo no necesariamente se centra en la persona que lo ostenta, si no que emerge de la interacción de los agentes del sistema organizacional y es necesario comprender cómo el poder que éste representa influye entre los seguidores-colaboradores, dado que se trata de un fenómeno relacional basado en la confianza otorgada a quien toma las decisiones que orienta el rumbo de la organización.

Tanto Terrazas (2015) como Contreras y Castro (2013b), basan sus 
estudios en características personales que debe tener el líder y la forma en que su posición es utilizada en la toma de decisiones, pero desde el punto de vista de los colaboradores, siendo ideal el autoanálisis bajo la percepción del mismo para identificar las limitantes propias para una adecuada toma de decisiones empresariales; lo anterior implica el desarrollo de estudios con diseño de instrumentos acordes a la propia visión del líder.

En el ámbito venezolano Loaiza y Pirela (2015), indagaron el estilo de liderazgo en las organizaciones de los sectores educativo y pymes industriales desarrollando una investigación teóricodocumental; sus hallazgos señalan una tendencia a requerir líderes orientados a la competitividad, con una visión que promueva la confianza y el optimismo en las compañías para emprender e innovar en la actividad económica en la que participan.

Baztar (2006), consideró importante proponer un modelo de liderazgo organizacional para las empresas mexicanas analizando la cultura y su impacto en la efectividad de las acciones de liderazgo, para lograr lo anterior estudió los conceptos relacionados con cultura organizacionalejercicio de liderazgo, evaluándolos a nivel nacional y latinoamericano considerando líneas de investigación teórico-metodológicas; y concluyó que aún se requieren modelos conceptuales y trabajo de campo no sesgado por la percepción del investigador, para explicar la vinculación o no de estas variables en el sector empresarial; estos estudios ratifican la necesidad formular estudios empíricos que no se limiten a recorridos teóricos sobre el tema, sino que indaguen sobre estos aspectos pero en actividades que son referente económicos como el sector construcción que se caracteriza por emplear a la población con menor preparación académica.

Se han desarrollado estudios empíricos sobre liderazgo transformacional utilizando instrumentos como los denominados Transformational Leadership Behavior Inventory (TLI) formulado por Podsakoff et al, (1996), y The Transformational Leadership Questionnaire (TLQ) diseñado por Alban-Metcalfe y Alimo-Metcalfe (2000).

En este sentido para obtener información sobre gestión del conocimiento, estrategias empresariales y liderazgo, Pedraja y Rodríguez (2008) Pedraja-Rejas, RodríguezPonce \& Rodríguez-Ponce (2009), analizaron su relación en pymes chilenas de diversos sectores mediante un cuestionario con escala tipo Likert integrado por seis variables (estilos transformacional, transaccional, crear conocimiento, compartirlo, aplicarlo y diseñar estrategias), cuya fiabilidad se obtuvo a través del coeficiente Alfa de Cronbach; estos autores utilizaron un modelo de regresión lineal para evaluar sus hipótesis, encontrando relaciones estadísticas significativas entre las variables abordadas donde los estilos de liderazgo tienen la capacidad explicativa del proceso de creación y aplicación del conocimiento en las organizaciones participantes y en el mismo sentido, la gestión del conocimiento influye en el diseño e implementación de estrategias, sin embargo no establecen si la percepción del líder incide en los procesos de gestión del conocimiento y cómo comparte esta visión con los colaboradores de la empresa.

Para analizar la influencia del liderazgo en la cultura organizacional Rodríguez (2010), utilizó un cuestionario 
cuya medida psicométrica fue el coeficiente Alfa de Cronbach para sus siete dimensiones: a) liderazgo transformacional, b) liderazgo transaccional, c) cultura de innovación, d) cultura competitiva, e) cultura burocrática, f) cultura comunitaria y g) eficacia; las cuales fueron validadas y utilizadas en investigaciones similares con índices de fiabilidad altos; los resultados que obtuvo el investigador se orientan hacia una relación entre los estilos de liderazgo y la cultura en sus modalidades de innovación y competitividad, lo que finalmente se refleja en la eficacia operacional de las empresas evaluadas, toda vez que los miembros de la organización asumen riesgos y desafíos para innovar y cumplir las metas asignadas lo que les permite crear un valor estratégico y económico, en este estudio el investigador realiza una mayor aproximación a la relación entre los valores personales y los organizacionales, lo que favorece identificar si la conducta del líder es acorde a los valores que forman parte de la cultura empresarial.

El liderazgo ejercido por los empresarios en el sector comercial de Tecomán, Colima y la percepción que tiene sobre el mismo el personal de las empresas fue abordado por Cárdenas et al, (2014), para lo cual utilizaron un cuestionario diseñado por Águila (2008), empleando la metodología de redes semánticas naturales establecieron que el estilo interpersonal tiene mayor prevalencia en estos líderes ya que no cuentan con un método específico de planeación del tiempo, que puede deberse a la falta de conocimiento sobre habilidades y características de un buen líder. Desde la perspectiva de los empleados son percibidos positivamente sin que se pueda afirmar que los conceptualicen como líderes verdaderos.

Sobre el liderazgo transformacional Hincapié et al, (2018), analizaron la influencia de los rasgos propios de este estilo sobre los procesos que desarrollan los equipos de trabajo en pymes colombianas, para lo cual diseñaron un instrumento para los constructos de liderazgo transformacional $y$ mejoramiento continuo, estableciendo de forma individual su confiabilidad mediante el Alfa de Cronbach y la validez de contenido a través del juicio de expertos; encontraron que la motivación inspiracional y la estimulación intelectual tienen mayor influencia positiva en las actividades de los grupos observados confirmando los resultados de estudios en otros contextos geográficos y culturales similares como Bogotá (Durán y Castañeda 2015), Cd. de México (Mendoza et al, 2014) y Caracas (Fernández y Quintero, 2017).

Uno de los instrumentos de medición de mayor aplicación en los estudios relacionados con el liderazgo es el cuestionario desarrollado por Kouzes y Posner (2003), y es identificado como Inventario de Prácticas de Liderazgo (IPL), el cual a través de procesos de evaluación y autoevaluación agrupados en cinco dimensiones establecen dos estilos, el primero de índole transformacional y el segundo de corte transaccional. Diversos autores han utilizado el coeficiente Alfa de Cronbach para establecer su nivel de confiabilidad interna, que generalmente se ubica en valores superiores a 0.75 para la versión de autoevaluación, aunque generalmente no se indica el procedimiento utilizado para determinar la validez del mismo. Este instrumento considera cinco prácticas fundamentales de liderazgo: a) Desafiar los procesos, 
donde los líderes aceptan retos y esperan al destino, b) Inspirar una visión compartida, es aquella donde los líderes albergan el deseo de transformar las cosas, c) Habilitar a los demás para que actúen, fomentando la colaboración y la confianza entre los colaboradores, d) Modelar el camino, esta dimensión pretende identificar la congruencia entre lo que dice el líder y lo que hace en el ámbito empresarial y e) Brindar aliento, en esta dimensión el objetivo es identificar si el líder estimula de forma positiva a sus colaboradores. Este cuestionario incluye treinta enunciados agrupados en las cinco dimensiones descritas y ha sido utilizado en estudios de organizaciones públicas y privadas.

En México, Robles et al,, (2008) analizaron las dimensiones de liderazgo en las pymes de los sectores industrial y comercial del estado de Tamaulipas a través del IPL, del cual establecieron su consistencia interna mediante el coeficiente Alfa de Cronbach, sin especificar pruebas de validez sobre el mismo. Los autores señalan que el número de personas bajo la dirección del gerente influye en las 5 dimensiones que incluye el IPL, y como dato particular refieren que los líderes de las empresas participantes requieren ser más innovadores en los procesos organizacionales ya que se comportan con base a una cultura nacional formada por creencias y valores del gerente por lo cual, no asumen riesgos ni comparten el poder. El liderazgo en el sector servicios de Tamaulipas, también fue sujeto de estudio por Vázquez y Pedraza (2014), quienes encontraron que las cinco dimensiones del IPL estuvieron presentes en las prácticas del liderazgo gerencial de las empresas, obteniendo la media más alta la dimensión denominada modelación del camino, pudiendo establecer que el estilo de liderazgo que prevalece en este sector es de tipo transformacional, en este caso se evaluaron las propiedades psicométricas del instrumento mediante el análisis factorial exploratorio (validez de constructo) y la fiabilidad con el Alfa de Cronbach.

En otro estudio sobre la práctica del liderazgo transformacional a través del IPL (Padilla et al, 2011), consideraron como población las pymes de los sectores comercial, industrial y de servicios del estado de Colima, sus hallazgos muestran que solo el 5.5\% de los líderes dirigen sus empresas con total responsabilidad de las mismas, sin contar con una estructura con áreas funcionales para su administración y operación, y consideran importante que el líder descubra su estilo, lo comprenda y lo depure ya que su actuación afectará a los colaboradores de la empresa.

En el ámbito del sector construcción existen factores que afectan la gestión del talento humano los cuales fueron estudiados por Torres (2018), en una muestra de profesionales expertos en administración de personal en obra civil en Colombia, con el ánimo de mejorar el nivel de bienestar de los operarios que laboran en este mercado y que se refleje en el incremento en los volúmenes de productividad. Sus resultados establecen que el trabajador debe enfrentar retos como la estabilidad laboral, poca motivación, exposición a riesgos físicos y psicológicos, por lo tanto, deben fomentarse esquemas de seguridad social benéficos para su bienestar, apostando a una capacitación que contribuya a la reducción de los accidentes laborales considerados propios de la actividad económica.

También sobre seguridad laboral Wu et al, (2017) consideraron que la 
frecuencia de accidentes se debe a la falta de liderazgo de los profesionales que colaboran en esta industria; los autores desarrollaron un modelo basado en perspectivas teóricas y pragmáticas, incorporando las características de los proyectos de construcción y niveles de gestión. El modelo de investigación-acción se utilizó para validar empíricamente el modelo teórico y poder desarrollar medidas factibles como reuniones fijas con el personal e incrementar la supervisión presencial de los líderes de los proyectos para implementar o reforzar la cultura sobre seguridad operativa y reducir las diferencias entre los equipos de trabajo. Los estudios formulados para el sector construcción por Torres (2018) y Wu et al, (2017), no establecen si los valores del líder y su visión del futuro empresarial inciden en los retos operativos que enfrentan los colaboradores y cómo afectan la gestión del talento humano en la organización.

\section{Petrovic (2002), investigó} los principales estilos de liderazgo que prevalecen en la industria de la construcción en Yugoslavia, al considerarla fundamental para el desarrollo económico de los países, ya que en este caso fue el último país socialista que migró a una economía de mercado. Los líderes de este sector tenían como principal objetivo el rápido enriquecimiento, pero tuvieron que enfrentar el periodo de transición de modelo económico de autogestión y la postura negativa en general de los trabajadores, de este proceso surgieron pymes que no podían cumplir contratos y recuperar su cartera. Ante la demanda del mercado los líderes de las organizaciones tuvieron que adoptar los estilos de gestión de las economías de libre mercado enfocadas en estrategias de alta gerencia para permanecer y evolucionar en el sector.

Newton (2008), examinó la discrepancia del concepto de liderazgo tradicional y la percepción del liderazgo adaptativo el cual se desarrolló como respuesta a los problemas sociales y ambientales y que se ha hecho presente en la industria de la construcción, donde se ha dado una confusión entre gestión y liderazgo. El autor concluye que se requieren líderes con capacidades gerenciales y gerentes con cualidades de liderazgo que emergen de un proceso de acción para un liderazgo adaptativo (cambio individual) que se haga presente en grupos de trabajo que generen valor en la industria; lo anterior refuerza la necesidad de establecer la incidencia de los valores personales y la verdadera inclusión de los equipos de colaboradores como parte del futuro organizacional, siempre desde la óptica del líder.

El liderazgo y el crecimiento de la industria de la construcción van de la mano en los países en desarrollo (Ofori y Toor, 2012) ya que las características de los proyectos y procesos los vinculan de forma esencial al crecimiento de las economías emergentes. Los autores consideran que el conocimiento sobre liderazgo auténtico abarca demasiados elementos positivos y es demasiado bueno para ser realista, sin embargo, es el concepto más adecuado a seguir por esta industria en los países emergentes, que requieren líderes auténticos, para alcanzar un nivel estratégico y desarrollo continuo.

La operacionalización del constructo del Liderazgo Dirigido (LD) para este estudio se basa en la conceptualización de una subescala del modelo de liderazgo de Kouzes y Posner (1996), quienes señalan que el liderazgo 
no es una posición, sino una colección de prácticas y comportamientos $y$ que forman parte del liderazgo transformacional (Abu-Tineh et al, 2008; Pariente, 2009). Este modelo se genera primordialmente a partir de la teoría de los rasgos y habilidades que debe tener un buen líder (Stodgill, 1950), y la teoría del camino - meta de House (1971).

\section{Consideraciones metodológicas}

La investigación presenta un enfoque cuantitativo con un diseño no experimental, transversal (Hernández et al, 2018), con la finalidad de generar un modelo de medida del constructo Liderazgo Dirigido (LD), que es una subescala de elaboración propia basada en la autopercepción del líder respecto a sus características y actitudes que dan coherencia a su conducta relacionada con los valores organizacionales y la visión compartida con los colaboradores sobre el futuro de la empresa.

El objetivo del estudio fue la validación psicométrica de esta subescala que se retomó del Inventario de Prácticas de Liderazgo de Kouzes y Posner (1996), así como la identificación de las diferencias entre los subsectores vivienda, educación, agua, comunicación, energía y especialidades.

Participaron 213 propietarios de empresas del sector construcción en el estado de Campeche situado en el sureste de México, todos ellos en este caso varones. El principal rango de edad $(30 \%)$ va de 53 años en adelante, y en su mayoría se encuentran casados (77.9\%). Un $69.5 \%$ posee estudios de educación superior y un $7.5 \%$ posgrado, siendo el nivel mínimo de estudio el bachillerato (8.2\%) o carrera comercial equivalente a este nivel (14.6\%). Se encuestaron compañías familiares $(30.5 \%)$, sociedades $(33.8 \%)$ y organizaciones con un solo propietario $(35.7 \%)$. En la tabla 1 se presenta una distribución de la población encuestada por subsector económico y por tipo de propiedad de la empresa.

\section{Tabla 1}

\section{Distribución de la población estudiada por subsector económico y tipo de propiedad de la organización.}

\begin{tabular}{lcccccc}
\hline \multirow{2}{*}{$\begin{array}{l}\text { Subsector } \\
\text { Económico }\end{array}$} & \multicolumn{2}{c}{ Único propietario } & \multicolumn{2}{c}{ Familiar } & \multicolumn{2}{c}{ De varios socios } \\
\cline { 2 - 7 } & $\mathrm{Fr}$ & $\%$ & $\mathrm{Fr}$ & $\%$ & $\mathrm{Fr}$ & $\%$ \\
\hline Vivienda & 27 & 35.5 & 5 & 7.7 & 26 & 36.1 \\
\hline Educación & 13 & 17.1 & 13 & 20.0 & 12 & 16.7 \\
\hline Agua & 1 & 1.3 & 7 & 10.8 & 0 & 0 \\
\hline Comunicación & 29 & 38.2 & 29 & 44.6 & 22 & 30.6 \\
\hline Energía & 0 & 0 & 7 & 10.8 & 9 & 12.5 \\
\hline Especialidades & 6 & 7.9 & 4 & 3.2 & 3 & 4.2 \\
\hline Total & 76 & $100 \%$ & 65 & $100 \%$ & 72 & $100 \%$ \\
\hline
\end{tabular}

Fuente: Elaboración propia (2020). 
Se solicitó a los participantes que contestaran el cuestionario de manera auto aplicado, a papel y lápiz, con acompañamiento de los miembros del equipo de investigación durante los meses de mayo a agosto del 2019.

El instrumento utilizado para obtener información se elaboró a partir de la versión en español del Inventario de Prácticas de Liderazgo de Kouzes y Posner (1996), desarrollada por Pedraza et al, (2014). Para su estructuración teórica se consultaron diversos estudios sobre el modelo de las cinco prácticas de liderazgo de Kouzes y Posner (Abu-Tineh et al, 2008; Pariente 2009; Pedraza et al, 2014), así como modelos de liderazgo basados en la teoría del camino-meta (Aalateeg, 2018; Alanazi et al, 2013; House, 1971; House y Aditya, 1997). Para la adaptación de los ítems se requirió una revisión literaria de otras escalas para la correcta redacción del contexto estudiado (Jun et al, 2011; Murdoch, 2013; Posner, 2016; Watters, 2019), lo que dio como resultado la escala que se propone y valida para esta investigación.

La validez de contenido se realizó de manera cualitativa con un grupo de expertos en liderazgo que a su vez fuesen autores de publicaciones sobre la temática general del tema abordado, a quienes se les pidió revisar los ítems de la escala para este constructo de acuerdo al procedimiento descrito por Escobar y Cuervo (2008) y Urrutia et al, (2015), para evaluar la evidencia empírica y los fundamentos teóricos de un instrumento.

El instrumento quedó conformado por cinco ítems que miden como primer factor la dimensión Guiar el camino (GC), definida como la autopercepción del líder que sirve de ejemplo de comportamiento coherente con los valores organizacionales; y cuatro para el segundo factor que mide la dimensión Visión Compartida (VC), definida como la autopercepción del líder que crea y comparte una visión positiva de futuro que atrae a los miembros de la organización (Kouzes \& Posner, 1996; Pedraza et al, 2014). La escala se estructuró tipo Likert con cuatro opciones de respuesta: 1: Muy en desacuerdo, 2: En desacuerdo, 3: De acuerdo, 4: Muy de acuerdo.

La fiabilidad de los datos obtenidos se estableció mediante el coeficiente Alpha de Cronbach y la validez del constructo y sus dimensiones se determinó a través de un análisis factorial exploratoria y confirmatoria. Por último, para identificar las diferencias entre los subsectores productivos se empleó la prueba de análisis unidireccional de varianza (ANOVA).

\section{Prácticas de liderazgo dirigido en pymes del sector construcción: Resultados}

Considerando que el objetivo del estudio fue la validación psicométrica de la subescala Liderazgo Dirigido y la identificación de las diferencias entre los subsectores en los que participan las empresas se verificaron las condiciones necesarias para el análisis factorial exploratorio (AFE), realizándose en primera instancia un estudio descriptivo que incluyó los valores de la media, desviación estándar, asimetría y curtosis de los ítems. Estos indicadores y la inspección visual del histograma permitieron establecer que los datos son simétricos respecto al promedio y su distribución se asemeja a una distribución normal (Tabla 2). 
Tabla 2

Media, Desviación Estándar, Asimetría y Curtosis de los reactivos de la escala Liderazgo Dirigido (LD).

\begin{tabular}{|c|c|c|c|c|}
\hline Ítems & M & $\mathrm{DE}$ & Asimetría & Curtosis \\
\hline \multicolumn{5}{|l|}{ Guiar el Camino } \\
\hline $\begin{array}{l}\text { Comunico con claridad la perspectiva positiva } \\
\text { para el futuro de nuestra organización. }\end{array}$ & 2.98 & .81 & -.49 & -.19 \\
\hline $\begin{array}{l}\text { Predico con el ejemplo en todas mis } \\
\text { actividades. }\end{array}$ & 3.03 & .65 & -.33 & .41 \\
\hline $\begin{array}{l}\text { Miro hacia adelante y visualizo cómo espero } \\
\text { que sea nuestro futuro. } \\
\text { Me muestro congruente con mi forma de }\end{array}$ & 2.99 & .72 & -.52 & .42 \\
\hline $\begin{array}{l}\text { actuar y lo que pienso acerca de lo que debe } \\
\text { ser el trabajo. }\end{array}$ & 3.02 & .78 & -.34 & -.52 \\
\hline $\begin{array}{l}\text { Cuestiono para mejorar nuestra manera de } \\
\text { hacer las cosas en la organización. }\end{array}$ & 2.76 & .88 & -.19 & -.73 \\
\hline \multicolumn{5}{|l|}{ Visión Compartida } \\
\hline $\begin{array}{l}\text { Tengo muy clara mi propia filosofía acerca del } \\
\text { liderazgo. }\end{array}$ & 2.94 & .90 & -.49 & -.56 \\
\hline $\begin{array}{l}\text { Busco maneras innovadoras de mejorar lo que } \\
\text { hacemos en nuestra organización. }\end{array}$ & 2.71 & .81 & -.21 & -.40 \\
\hline $\begin{array}{l}\text { Describo a los demás el futuro que me } \\
\text { gustaría que labráramos juntos. }\end{array}$ & 2.85 & .87 & -.28 & -.70 \\
\hline $\begin{array}{l}\text { Busco oportunidades de reto que pongan a } \\
\text { prueba mis aptitudes. }\end{array}$ & 2.66 & .94 & -.17 & -.87 \\
\hline
\end{tabular}

Fuente: Elaboración propia (2020)

La homogeneidad y consistencia interna del instrumento se aseguró mediante el cálculo de los valores del Alfa de Cronbach, obteniendo un nivel de confiabilidad general de $\alpha=0.70$ (GC: $\alpha=0.66$ y VC: $\alpha=0.60$ ) que puede considerarse como aceptable y confiable para el estudio de las dos dimensiones que integran el instrumento de liderazgo dirigido (Quero, 2010). Para estar en condiciones de validar el constructo de la escala también se verificaron los supuestos para el análisis multivariante a través de la prueba de Kaiser-MeyerOlkin (KMO) y la prueba de esfericidad de Bartlett $(\mathrm{KMO}=.73, \mathrm{gl}=36, \mathrm{c} 2=278.77$, $\mathrm{p}<0.000)$, cuyos resultados indican que se puede proceder a desarrollar el Análisis Factorial Exploratorio (AFE) (Williams et al, 2010).
Se realizó el AFE mediante el método de extracción de factores por máxima verosimilitud con rotación oblimin directo para dar evidencia de la validez de constructo de la escala, e identificar el número y composición de los factores comunes necesarios para explicar la varianza común del conjunto de ítems analizados cinco para la dimensión GC y cuatro para VC. En los resultados del AFE se observa que las cargas factoriales son superiores a 0.3 cumpliéndose así los criterios mínimos para mantener todos los ítems (Williams et al, 2010), sin embargo los valores de las comunalidades para algunos de ellos son relativamente bajas como se observa en la tabla 3. 


\section{Tabla 3}

Análisis factorial de la escala Liderazgo Dirigido (LD)

\begin{tabular}{|c|c|c|c|}
\hline Ítem & Factor 1 & Factor 2 & $h^{2}$ \\
\hline \multirow{6}{*}{$\begin{array}{l}\text { Guiar el Camino } \\
\text { Comunico con claridad la perspectiva positiva para el futuro } \\
\text { de nuestra organización. } \\
\text { Predico con el ejemplo en todas mis actividades. } \\
\text { Miro hacia adelante y visualizo como espero que sea } \\
\text { nuestro futuro. } \\
\text { Me muestro congruente con mi forma de actuar y lo que } \\
\text { pienso acerca de lo que debe ser el trabajo. } \\
\text { Cuestiono para mejorar nuestra manera de hacer las cosas } \\
\text { en la organización. }\end{array}$} & & & \\
\hline & .67 & & .29 \\
\hline & .55 & & .23 \\
\hline & .54 & & .40 \\
\hline & .46 & & .41 \\
\hline & .30 & & .38 \\
\hline \multicolumn{4}{|l|}{ Visión Compartida } \\
\hline Tengo muy clara mi propia filosofía acerca del liderazgo. & & .63 & .33 \\
\hline $\begin{array}{l}\text { Busco maneras innovadoras de mejorar lo que hacemos en } \\
\text { nuestra organización. }\end{array}$ & & .57 & .28 \\
\hline $\begin{array}{l}\text { Describo a los demás el futuro que me gustaría que } \\
\text { labráramos juntos. }\end{array}$ & & .45 & .17 \\
\hline $\begin{array}{l}\text { Busco oportunidades de reto que pongan a prueba mis } \\
\text { aptitudes. }\end{array}$ & & .31 & .18 \\
\hline$\%$ de varianza total explicada $=30.14 \%$ & & & \\
\hline
\end{tabular}

Nota: $\mathrm{N}=213, \mathrm{KMO}=0.73, g l=36, \mathrm{c}^{2}=278.77, p<0.000, h^{2=}$ Comunalidad. Método de extracción: Análisis de máxima verosimilitud con rotación oblimin directo. Factor 1 = Guiar el Camino (GC), Factor 2: Visión Compartida (VC).

Fuente: Elaboración propia (2020)

Batista-Foguet et al, (2004) y Fernández (2015), hacen una crítica severa a los métodos de validación de constructo únicamente a través del análisis factorial exploratorio y proponen como un complemento necesario establecer modelos de medida generados a través del Análisis Factorial Confirmatorio (AFC). Estos modelos permiten establecer la validez considerando la bondad de ajuste, en donde cada ítem satura únicamente sobre el factor que constituye un indicador válido. Para este modelo teórico se realizó un Análisis Factorial Confirmatorio (AFC) que permitió demostrar las cargas factoriales, la validez discriminante y convergente para la estructura previamente obtenida, y de las deducciones teóricas inferidas en él (Littlewood \& Bernal, 2014).
De acuerdo con Arias (2008) y Manzano y Zamora (2010), se calcularon los principales indicadores de ajuste del modelo. En primer término, se presentan los indicadores del grado de bondad de ajuste: la prueba chi cuadrada $\left(X^{2}\right)$ y la razón de chi cuadrada sobre los grados de libertad; posteriormente se muestran los índices de ajuste absolutos: la raíz media cuadrada de los residuos (SRMR), el índice ajustado de bondad de ajuste (AGFI) y el índice de aproximación de la raíz de cuadrados medios del error (RMSEA), los cuales muestran valores aceptables. Por último, se reportan los valores de los principales índices de ajuste de incremento: el índice de ajuste no normado (TLI), y el índice de ajuste comparativo (CFI), que también obtuvieron valores satisfactorios (Tabla 4). En el modelo de medida se puede 
pp. $492-509$

Revista Venezolana de Gerencia, Año 26 No. 94, 2021

apreciar cargas factoriales significativas, por encima de 0.60 , en todos los ítems de ambos factores; por su parte la covarianza entre las variables del modelo resulta positivamente significativa (diagrama 1).

\section{Tabla 4}

\section{Indicadores de ajuste de modelo estructural de la escala Liderazgo Dirigido (LD).}

\begin{tabular}{|c|c|c|c|c|c|}
\hline & \multicolumn{5}{|c|}{ Indicadores del grado de bondad de ajuste del modelo } \\
\hline Indicador & $x^{2}$ & $g l$ & $\mathrm{p}$ & & $/ \mathrm{df}$ \\
\hline Valores esperados & & & $>0.001$ & & a 3 \\
\hline Modelo & 35.53 & 24 & 0.061 & & 48 \\
\hline & \multicolumn{3}{|c|}{ Índices de ajuste absoluto } & \multicolumn{2}{|c|}{$\begin{array}{l}\text { Indices de ajuste de } \\
\text { incremento }\end{array}$} \\
\hline Indicador & SRMR & RMSEA & AGFI & TLI & $\mathrm{CFI}$ \\
\hline Valores esperados & $<0.08$ & 0.06 a 0.08 & $\geq 0.90$ & $\geq 0.90$ & $\geq 0.95$ \\
\hline Modelo & 0.03 & 0.04 IC $90[.00-.07]$ & 0.93 & 0.93 & 0.95 \\
\hline
\end{tabular}

Nota: $\quad \mathrm{N}=2$ 213. Valores de referencia tomados de: Arias, 2008; Manzano y Zamora, 2010.

Fuente: Elaboración propia (2020)

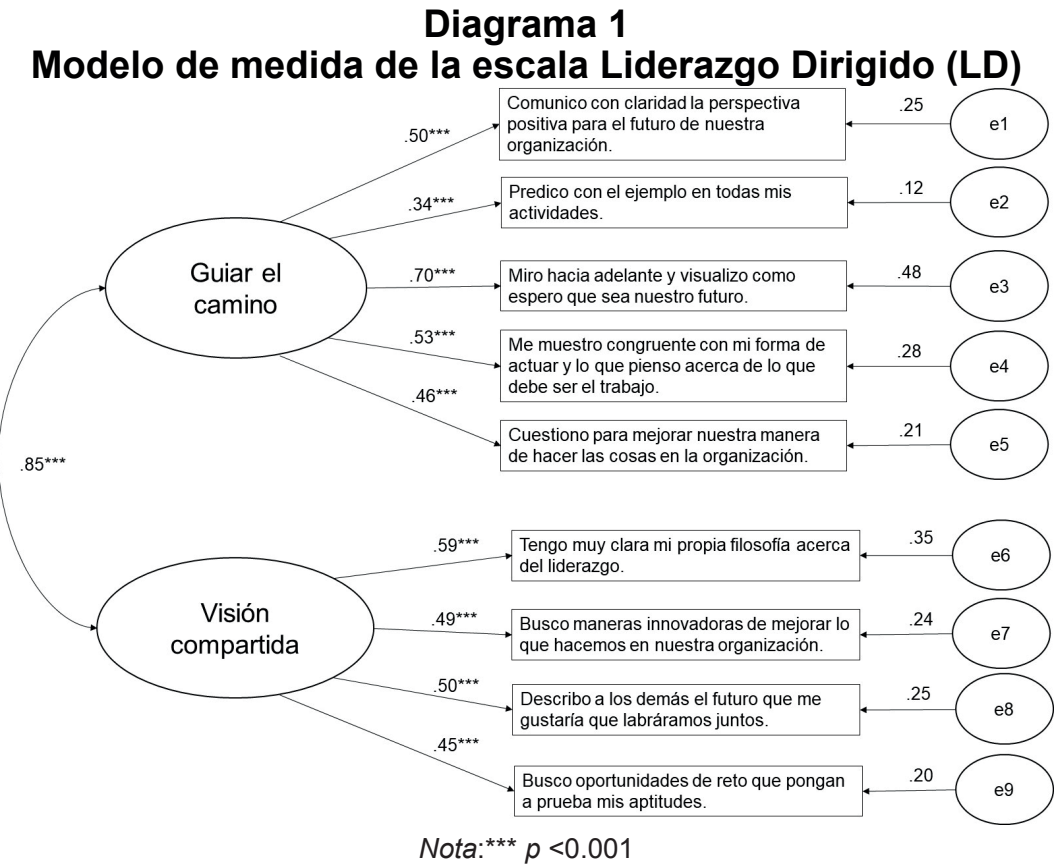

Fuente: Elaboración propia (2020) 
El modelo cumple con presentar cinco variables observadas en el factor Guiar el camino y cuatro para el factor Visión compartida con cargas factoriales superiores a 0.6 lo cual es aceptable (Littlewood \& Bernal, 2014; Manzano \& Zamora, 2010). Batista-Foguet et al,, (2004), señalan que el modelo de análisis factorial confirmatorio permite corregir deficiencias presentadas en el exploratorio y a su vez analizar la matriz de covarianzas y no solo la de correlaciones, lo que para este modelo se hacía necesario dado los valores de algunas de las comunalidades (Kano \&
Ihara, 1994; Mulaik \& Millsap, 2009). El empleo de ambas técnicas estadísticas otorga un mayor rigor al proceso de desarrollo de la escala.

Una vez establecidas las características de fiabilidad y validez del constructo, se pueden determinar las diferencias que están presentes en el liderazgo dirigido por subsector económico (Tabla 5). Los valores reportados señalan diferencias significativas en las dos dimensiones que integran las prácticas de liderazgo dirigido, las cuales pueden explicarse por el tipo de sector económico.

\section{Tabla 5}

\section{Media, Desviación Estándar, Análisis Unidireccional de Varianza} entre las Dimensiones de Liderazgo Dirigido

\begin{tabular}{|c|c|c|c|c|c|c|c|c|c|c|c|c|c|c|}
\hline \multirow{2}{*}{ Dimensiones } & \multicolumn{2}{|c|}{1} & \multicolumn{2}{|c|}{2} & \multicolumn{2}{|c|}{3} & \multicolumn{2}{|c|}{4} & \multicolumn{2}{|c|}{5} & \multicolumn{2}{|c|}{6} & \multirow{2}{*}{$F$} & \multirow{2}{*}{$\eta^{2}$} \\
\hline & $M$ & $\mathrm{DE}$ & $M$ & DE & $M$ & DE & $M$ & $\mathrm{DE}$ & $M$ & $\mathrm{DE}$ & $M$ & $\mathrm{DE}$ & & \\
\hline $\begin{array}{l}\text { Guiar el camino } \\
\text { (GC) }\end{array}$ & 3.03 & .41 & 3.12 & .41 & 3.55 & .48 & 2.77 & .41 & 2.53 & .80 & 3.4 & .24 & $12.17^{\star \star \star \star}$ & 0.23 \\
\hline $\begin{array}{l}\text { Visión compartida } \\
\text { (VC) }\end{array}$ & 3.10 & .49 & 3.23 & .29 & 3.15 & .39 & 2.35 & .52 & 2.59 & .39 & 2.73 & .34 & $27.99^{* \star \star}$ & 0.40 \\
\hline
\end{tabular}

Nota: Sectores productivos: 1= vivienda, 2= Educación, 3= Agua, 4= Comunicación, 5= energía, 6= Especialidades.

${ }^{*} p<.05 ;{ }^{* *} p<.015 ;{ }^{* * *} p<.001$

Fuente: Elaboración propia (2020)

Los resultados que se presentan del tamaño del efecto indican que el $23 \%$ de las diferencias en la dimensión guiar el camino, y $40 \%$ en la visión compartida, pueden ser explicadas por el tipo de subsector al que pertenece la empresa constructora, y por ello es importante identificar aquellos grupos que si son diferentes (Tejero et al, 2012). Para ello los resultados de la prueba post hoc de Tukey relativa a la dimensión guiar el camino, reporta que las empresas de construcción que pertenecen a subsector del agua presentan valores significativamente superiores que las demás áreas, con excepción al sector de vivienda, que no presentó significancia estadística en esta prueba, siendo el valor más pequeño el que reporta el sector de energía. Para la segunda dimensión, visión compartida, los resultados de la prueba post hoc de Tukey muestran diferencias significativas únicamente para los subsectores de vivienda, comunicación y energía, siguiendo esta misma jerarquía para los valores 
reportados. En los demás subsectores no se observaron diferencias para esta dimensión.

\section{Conclusiones}

Esta investigación planteó un modelo que es una subescala del Inventario de Prácticas de Liderazgo de Kouzes \& Posner (1996) al cual se le denominó Liderazgo Dirigido conceptualizado como la autopercepción del líder respecto a sus características y actitudes que dan coherencia a su conducta relacionada con los valores organizacionales y la visión compartida con los colaboradores sobre el futuro de la empresa.

Las empresas participantes
pertenecen al sector construcción que se caracteriza por ser indicador del comportamiento de la economía de un país al proporcionar empleo a la población con menor preparación académica y oportunidades laborales, lo que justifica la pertinencia social de este estudio. Se estructuró un instrumento integrado por dos dimensiones Guiar el Camino y Visión Compartida, y cuyas propiedades psicométricas de homogeneidad y consistencia interna se establecieron mediante el coeficiente Alfa de Cronbach. Con la técnica del juicio de expertos se verificó la validez de contenido y mediante el análisis factorial exploratorio y confirmatorio se estableció la validez de constructo para dar consistencia a las conclusiones sobre los resultados de la investigación.

Este trabajo se suma al esfuerzo de estructurar instrumentos cuya validez y confiabilidad sean evaluadas mediante procesos estadísticos rigurosos, que contribuyen a la obtención de información sobre la causalidad y validar hipótesis teóricas previamente establecidas relacionadas con la autoevaluación de la personalidad del líder y su influencia en toma de decisiones empresariales según su ámbito sectorial y geográfico. $\mathrm{Si}$ bien el estudio no realiza aportes metodológicos a la técnica estadística utilizada, si se logra el objetivo de verificación de elementos necesarios en psicometría para considerar adecuado un modelo de medida.

Como limitantes, se puede mencionar la validez externa derivada de la circunscripción geográfica y el número de unidades económicas participantes; por lo cual la réplica de los procedimientos desarrollados en otros ámbitos y sectores económicos puede generar futuras líneas de investigación para evaluar aquellas características del liderazgo y las prácticas que inciden en la forma en que el empresario toma las decisiones, demostrando su compromiso con los valores organizacionales y la congruencia entre lo que propone y finalmente realiza.

\section{Referencias bibliográficas}

Aalateeg, S. (2018). Literature Review on Leadership Theories. International Journal of Research in Business Management (IOSR-JBM), 19(11), 35-43. https://iosrjournals.org/iosrjbm/papers/Vol19-issue11/Version-3/ E1911033543.pdf

Abu-Tineh, A., Khasawneh, S., \& AlOmari, A. (2008). Kouzes and Posner's transformational leadership model in practice: The case of Jordanian schools. Leadership \& Organization Development Journal, 29(8), pp. 648-660. http://doi. org/10.1108/01437730810916613

Alanazi, R., Alharthey, K., \& Amran, 
Quijano García, Román Alberto; Magaña Medina, Deneb Elí

Prácticas de liderazgo dirigido en pymes del sector construcción. Propiedades

psicométricas en la medición

R. (2013). Overview of path-goal leadership theory. Jurnal Teknologi (Sciences and Engineering), 64(2), 49-53. http://doi.org/10.11113/ jt.v64.2235

Alban-Metcalfe, R., y Alimo-Metcalfe, B. (2000). The transformational leadership questionnaire (TLQLGV): a convergent and discriminant validation study. Leadership \& Organization Development Journal, 21(6), 280-296. http:// www.emerald.com/insight/content/ doi/10.1108/01437730010343077/ full/html

Arias, B. (2008). Desarrollo de un ejemplo de análisis factorial confirmatorio con LISREL, AMOS y SAS: Seminario de Actualización en Investigación sobre Discapacidad SAID, 1-42.

Batista-Foguet, J., Coenders, G., \& Alonso, J. (2004). Confirmatory factor analysis. Its role on the validation of health related questionnaires. Medicina Clínica (Barcelona), 122(1), 21-27. https://bit.ly/2Rhn3KY

Baztar, S. (2006). Reflexiones iniciales para la construcción de un modelo mexicano de liderazgo organizacional. Revista de Psicología de Trabajo y de las Organizaciones, 22(2), 203-225. http://www.redalyc. org/articulo.oa? id=231317125004

Cárdenas, A., Lino, J., y Macías, E. (2014) Liderazgo ejercido por los empresarios comerciales de la zona centro de Tecomán, Colima, México. Memorias en extenso del XVIII Congreso Internacional de Investigación en Ciencias Administrativas (ACACIA), 1, 53115339 .

Centro de Estudios Económicos del Sector de la Construcción (2020). Situación de la actividad productiva de las empresas constructoras. www. cmic.org.mx
Contreras, F., y Barbosa, D. (2013a). Del liderazgo transaccional al liderazgo transformacional: implicaciones para el cambio organizacional. Revista Virtual Universidad Católica del Norte, 39, 152-164. http://www. redalyc.org/pdf/1942/194227509013. pdf

Contreras, F., y Castro, G. (2013b). Liderazgo, poder y movilización organizacional. Estudios Gerenciales, 29(126), 72-76. http://www.redalyc. org/pdf/212/21228397008.pdf

Contreras, F., y Juárez, F. (2013c). Efecto del capital psicológico sobre las prácticas de liderazgo en PYMES colombianas. Revista Venezolana de Gerencia (RVG), 18(62), 247264. https://www.redalyc.org/articulo. oa? id=29026923007

Durán, M., y Castañeda, D. (2015). Relación entre liderazgo transformacional y transaccional con la conducta de compartir conocimiento en dos empresas de servicios. Acta Colombiana de Psicología. 18(1), 135-147. http://www.redalyc.org/articulo. oa? id=79838614013

Escobar-Pérez, J. \& Cuervo-Martínez, Á. (2008). Validez de contenido y juicio de expertos: Una aproximación a su utilización. Avances en Medición, 6, 27-36. https://bit.ly/3uAcssQ

Fernández, A. (2015). Aplicación del análisis factorial confirmatorio a un modelo de medición del rendimiento académico en lectura. Ciencias Económicas, 33(2), pp. 39-66. https:// bit.ly/3s71rxQ

Fernández, C., y Quintero, N. (2017). Liderazgo transformacional $y$ transaccional en emprendedores venezolanos. Revista Venezolana de Gerencia (RVG), 22(77), pp. 5674. http://www.redalyc.org/articulo. oa?id=29051457005 
Gobierno del Estado de Campeche. (2020). Plan Estatal de Desarrollo 2019-2021. http://www.seplan. campeche.gob.mx/index.php/ noticias-info/286-presentacion-delplan-estatal-de-desarrollo-2019-2021

Gobierno Federal de los Estados Unidos Mexicanos, Presidencia de la República. (2020). Plan Nacional de Desarrollo 2019-2024. México. https://observatorioplanificacion. cepal.org/es/planes/plan-nacionalde-desarrollo-de-mexico-2019-2024

Hernández, R., Fernández, C., y Baptista, P. (2018). Metodología de la investigación. Mc Graw Hill.

Hincapié, S., Zuloaga, Y., y López, E. (2018). Liderazgo transformacional y mejoramiento continuo en equipos de trabajo de pymes colombianas. Revista Venezolana de Gerencia (RVG), 23(83), 1-17. https://bit. Iy/3tb1gTa

House, R. (1971). A path goal theory of leader effectiveness. Administrative Science Quarterly, 16(3), 321$339 . \quad$ https://www.jstor.org/ stable/2391905?seq=1\#page scan tab contents

House, R., \& Aditya, R. (1997). The social scientific study of leadership: Quo vadis? Journal of Management, 23(3), 409-473. http://doi. org/10.1177/014920639702300306

Instituto Nacional de Estadística y Geografía (2020). Encuesta Nacional de Ocupación y Empleo. https://www.inegi.org.mx/programas/ enoe/15ymas/

Instituto Nacional de Estadística y Geografía (2020). PIB y Cuentas Nacionales. https://www.inegi.org. mx/temas/pib/

Jun, L., Xiaoyu, L., \& Xianju, Z. (2011). Does transactional leadership count for team innovativeness? Journal of Organizational Change Management, 24(3), 282-298. https://doi.org/http://dx.doi. org/10.1108/09534811111132695

Kano, Y., \& Ihara, M. (1994). Identification of inconsistent variates in factor analysis. Psychometrika, 59(1), 5-20. https://doi.org/10.1007/BF02294262

Kouzes, J., y Posner, B. (2003). The Leadership Practices Inventory (LPI): Self Instrument. John Wiley \& Sons Inc. U.S.A.

Kouzes, James \& Posner, Barry. (1996). The leadership challenge: How to keep getting extraordinary things done in organizations. The Leadership Quarterly, 7(3), pp. 427. https://doi.org/10.1016/s10489843(96)90028-4

Littlewood, H., \& Bernal, E. (2014). Mi primer modelamiento de ecuaciones estructurales, 2a edición.

Loaiza, C., y Pirela, L. (2015). Liderazgo en organismos venezolanos. Revista Venezolana de Gerencia (RVG), 20(69), 152-171. https://www.redalyc. org/pdf/290/29036968009.pdf

Manzano, A., \& Zamora, S. (2010). Sistema de ecuaciones estructurales:una herramienta de investigación. Cuaderno técnico 4. México: Centro Nacional de Evaluación para la Educación Superior, (CENEVAL).

Mendoza, I., García, B., y Uribe, J. (2014). Liderazgo y su relación con variables de resultado: un modelo estructural comparativo entre liderazgo transformacional $y$ transaccional en una empresa de entretenimiento en México. Acta de Investigación Psicológica, 4(1), 1412-1429. https://www.scielo.org. mx/pdf/aip/v4n1/v4n1a8.pdf 
Quijano García, Román Alberto; Magaña Medina, Deneb Elí

Prácticas de liderazgo dirigido en pymes del sector construcción. Propiedades

psicométricas en la medición

Mulaik, S., \& Millsap, R. (2009). Structural Equation Modeling: A Doing the Four-Step Right. Structural Equation Modeling: A Multidisciplinary Journal, 7(1), 36-73. https://doi.org/10.1207/ S15328007SEM0701 02

Murdoch, L. (2013). An Investigation of Path-Goal Theory , Relationship of Leadership Style, SupervisorRelated Commitment, and Gender. Emerging Leadership Journeys, 6(1), 13-44.

Newton, S. (2008). Changing the framework for leadership in the construction industry. $24^{\text {th }}$. Annual ARCOM Conference, 433-442

Ofori, G., y Toor, Shamas-ur-Rehman. (2012). Leadership and construction industry development in developing countries. Journal of Construction in Developing Countries, 17, 1-21. https://bit.ly/2QesFpo

Padilla, A., Hernández, L., y Espíritu, R. (2011). La Práctica del Liderazgo Transformacional: Un estudio empírico realizado en las pymes en el estado de Colima, México. Revista Universitaria Digital de Ciencias Sociales (RUDICS), 1-15. https://virtual.cuautitlan.unam.mx/ rudics/?p=343

Pariente, J. (2009). Algunas reflexiones en torno al concepto del liderazgo. Proceso de Cambio y Desarrollo Organizacional, 153-189.

Pedraja, L., y Rodríguez, E. (2008). Estilo de liderazgo, gestión de conocimiento y diseño de la estrategia: un estudio empírico en pequeñas y medianas empresas. Interciencia, 33(9), 651657. https://bit.ly/3dSc6sm

Pedraja-Rejas, L., RodríguezPonce, E., \& Rodríguez-Ponce, J. (2009). Importancia de los estilos de liderazgo sobre la eficacia: un estudio comparativo entre grandes y pequeñas y medianas empresas privadas. Revista De Ciencias Sociales, 14(1). https://doi. org/10.31876/rcs.v14i1.25384

Pedraza, N., Lavín, J., Delgado, G., y Bernal,I. (2014). Prácticas de liderazgo en empresas comerciales en Tamaulipas (México). Revista Facultad de Ciencias Económicas, 23(1), 251-265. https://doi. org/10.18359/rfce.618

Petrovic, S. (2002). Challenges of leadership in Yugoslav building construction industry. Working paper series Monash University, Faculty of Business and economics, 1-8

Podsakoff, P., Mackenzie, S., \& Bommer, W. (1996). Transformational leader behaviors and substitutes for leadership as determinants of employee satisfaction, commitment, trust, and organizational citizenship behaviors. Journal of Management. 22(2), 259-298. https://www.researchgate.net/ publication/270726799

Posner, B. (2016). Investigating the Reliability and Validity of the Leadership Practices Inventory®. Administrative Sciences, 6(4), $17 . \quad$ https://doi.org/10.3390/ admsci6040017

Quero, M. (2010). Confiabilidad y coeficiente Alpha de Cronbach. Telos, 12(2), 248-252. https://www. redalyc.org/pdf/993/99315569010. pdf

Robles, V., de la Garza, M., y Medina, J. (2008). El liderazgo de los gerentes de las pymes de Tamaulipas, México. Cuadernos de Administración, 21(37), 293-210. https://www.redalyc. org/pdf/205/20503713.pdf

Rodríguez, E. (2010). Estilos de liderazgo, cultura organizacional y eficacia. Revista de Ciencias Sociales, XVI(4), 
629-641. https://www.redalyc.org/ articulo.oa?id=28016613006

Stodgill, R. (1950). Leadership, membership, and organization. Psychological Bulletin, 47(1), 1-14. https://www.ncbi.nlm.nih.gov/ pubmed/19586159

Tejero-González, C., Castro-Morera, M., y Balsalobre-Fernández, C. (2012). Importancia del tamaño del efecto. Una ejemplificación estadística con medidas de condición física. Revista Internacional de Medicina y Ciencias de la Actividad Física y el Deporte, 12(48), 715-727. https:// cdeporte.rediris.es/revista/revista48/ artimportancia318.htm

Terrazas, Rafael. (2015). Liderazgo tridimensional. Revista Perspectivas, 35, pp. 7-28.

Torres, Edward. (2018). Aspectos que afectan la gestion del talento humano en el sector construcción en Colombia. Signos, 10(2), pp.103$117 . \quad$ https://www.redalyc.org/ jatsRepo/5604/560459866006/html/ index.html

Turbay-Posada, M. (2013). Liderazgo e innovación organizacional. Psicología desde el Caribe, 30(1), 7-9. https://www.redalyc.org/articulo. oa?id=21328600001

Urrutia, M., Barrios, S., Gutiérrez, M., \& Mayorga, M. (2015). Métodos óptimos para determinar validez de contenido. Revista Cubana de Educación Médica Superior, 28(3), 547-558.

Vázquez, M., y Pedraza, N. (2014) Las prácticas de liderazgo en las mipymes del sector servicios en Cd. Victoria,
Tamaulipas, México. XVIII Congreso Internacional de Investigación en Ciencias Administrativas (ACACIA). Memorias en Extenso, 5280-5310.

Velázquez, G. (2005). Liderazgo empático "Un modelo de liderazgo para las organizaciones mexicanas". Revista del Centro de Investigación, 6(23), 81-100. https://www.redalyc. org/pdf/342/34202307.pdf

Watters, Eric. (2019). The applicability of Kouzes and Posner's leadership practices inventory in measuring the use of transformational leadership practices in law enforcement: A review of the literature. Forensic Science and Crime Reseach, 1(1), 1-5. https://bit.ly/3dWRYEh

Williams, B., Onsman, A., \& Brown, T. (2010). Exploratory factor analysis: A five-step guide for novices. Journal of Emergency Primary Health Care (JEPHC), 8(3), 1-13. https://ajp. paramedics.org/index.php/ajp/article/ view/93/90

Wu, Ch., Li, N., y Fang, D. (2017). Leadership improvement and its impact workplace safety in construction projects: a conceptual model and action research. International Journal of Project Management. 35, 1495-1511. https://dx.doi.org/10.1016/j. ijproman.2017.08.013

Zayas, P. (2011). El desempeño, el liderazgo y las competencias en los directivos del sector turístico. Revista de investigación en turismo $y$ desarrollo local (TURyDES), 4(11). https://www.eumed.net/rev/ turydes/11/pmza.html 